\title{
Parents' perceptions of autism and their health-seeking behaviors
}

\author{
Maria Isabel O. Quilendrino ${ }^{a, *}$, Mary Anne R. Castor ${ }^{a}$, \\ Nenacia Ranali Nirena P. Mendoza ${ }^{b}$, Jacqueline R. Vea ${ }^{c}$, \\ Nina T. Castillo-Carandang ${ }^{c}$

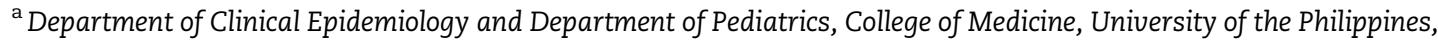 \\ Manila, Philippines \\ ${ }^{\mathrm{b}}$ Department of Clinical Epidemiology and Department of Family and Community Medicine, College of Medicine, \\ University of the Philippines, Manila, Philippines \\ ${ }^{\mathrm{c}}$ Department of Clinical Epidemiology, College of Medicine, University of the Philippines, Manila, Philippines
}

\section{A R T I C L E I N F O}

Article history:

Received 15 September 2015

Accepted 13 November 2015

Available online 7 December 2015

Keywords:

Autism

Autistic disorder

Behavior

Perception

\begin{abstract}
A B S T R A C T
Background: Parents' health-seeking behaviors, which in turn may be related to their perceptions, are hypothesized to be the major determinant of the timing of diagnosis and subsequent intervention for children with autism.

Objective: The primary objective of this study was to describe parental perceptions of autism and health-seeking behaviors for urban Filipino children aged 2-6 years.

Methods: This was a cross-sectional study conducted in several phases. The first phase involved collection of qualitative data from key informant interviews and small group discussions. The second phase involved the development of a validated and reliable questionnaire, which was administered to 41 parents of children with autism, aged 2-6 years. Results: Parents had varying perceptions of autism. They were generally undecided with regard to the etiology of autism, but were in agreement that psychosocial factors, such as parental sins and curses, were unlikely to be associated with autism. The most common presenting symptom noted by parents was a qualitative impairment in social interaction. There was a noted trend towards earlier age of symptom recognition (mean of 24 months) and diagnosis of autism (mean of 39 months) among parents with younger children.

Conclusion: The results of this study showed some trends: There is improved awareness regarding autism and the needs of children with autism. Parents tend to disagree with previous myths about autism. There was an observable trend toward earlier diagnosis for this group.
\end{abstract}

(C) 2015 INDIACLEN. Published by Elsevier, a division of Reed Elsevier India, Pvt. Ltd. All rights reserved.

\footnotetext{
* Corresponding author. Tel.: +63 9178033888; fax: +63 27311631.

E-mail address: iosabel@yahoo.com (M.I.O. Quilendrino).

http://dx.doi.org/10.1016/j.cegh.2015.11.003
}

2213-3984/@ 2015 INDIACLEN. Published by Elsevier, a division of Reed Elsevier India, Pvt. Ltd. All rights reserved. 


\section{Introduction}

It can be said that autism is no longer a rare disorder, yet at present, it is still incompletely understood. A 5-year review of developmental referrals to a tertiary government hospital in Manila showed that autism ranked second, next to global developmental delay and mental retardation, as the top diagnoses for referrals. ${ }^{1}$

The increasing prevalence of autism may be reflective of either a true increase in the number of cases or any of the following: increased societal awareness, changing diagnostic criteria, and better access to educational services. ${ }^{2}$ Presenting problems of the disorder vary greatly depending on the developmental level and chronological age of the individual. ${ }^{3}$ Although early identification is encouraged by healthcare professionals, a significant lag between age at first parental concern and age at first ASD diagnosis is consistently reported in the literature. ${ }^{4}$ While much about autism is unclear, the importance of early intervention for such children is almost universally acknowledged. Early detection and intervention are critical in altering the disabling trajectory of autism. ${ }^{1}$

Parents are usually the first to recognize signs of autism. Parents' perceptions and beliefs may drive their health behaviors - whether to seek formal evaluation or choose to wait. Recognition of a problem is the first step of the process. ${ }^{5}$ Researches show that early signs of autism were reported as early as 1 year of age $^{6}$ and that autism can be reliably diagnosed by 2 years of age, and yet the mean age of diagnosis for autism is generally delayed. A study done in the Neurodevelopmental Section of the Philippine Children's Medical Center showed that the mean age of recognition is 1.8 years and the interval between the onset of symptoms and referral is 2.6 years. ${ }^{7}$ The most common manifesting symptom and reason for referral was language delay.

Parents' health-seeking behavior is an essential driving force in determining the length of this period. Understanding the effect of parental perceptions on their health-seeking behavior would aid in developing targeted interventions aimed at addressing these perceptions in order to modify behavior geared towards shortening the period from symptom recognition to formal diagnosis.

A local study on factors implicated in the delayed consultation of children with neurodevelopmental disabilities cited parental factors as a main reason for delays in consultation. These parental factors were related to parents' beliefs or hope that their children will eventually catch up, general lack of knowledge regarding normal child development, and lack of funds. ${ }^{8}$

An analysis of a large sample of US children born in 1994 found that being male, having a low IQ and experiencing developmental regression were all associated with earlier diagnosis for children. ${ }^{9}$ Children with more educated parents were diagnosed earlier. Having a mother born outside the USA and being first born were also associated with later diagnoses. Children with higher communication function were diagnosed much later than those with lower function. ${ }^{8}$ A local study in an urban low income community in Manila showed that parents' educational level affected the rate of reporting of developmental concerns, and that parents were more tolerant of delays in language development when the child was either a male or less than 2 years of age. ${ }^{10}$

Parents cited a range of explanations to account for their son's or daughter's disability, including birth trauma and illness during pregnancy. Some parents also expressed beliefs that do not conform to biomedical explanations, but drew on magical or religious beliefs. A sense of guilt was featured in many parents' descriptions. Some parents believed that autism was a punishment for past mistakes, whereas parents who ascribed to a biomedical explanation of autism perceived a responsibility for passing on faulty genes to their child with autism. Gray concluded that these explanatory models enabled parents to make sense of autism and facilitated their ability to cope. ${ }^{11}$ Parental views regarding the causes of autism are shown to affect their health practices for their children. The view that vaccines (thimerosal; measles, mumps rubella [MMR] vaccines) were behind the autism issue led to mistrust of physicians, a decrease in vaccination rates, and an increase in antivaccine movements in many countries, leading to outbreaks of vaccine-preventable diseases. ${ }^{12}$

Parental perceptions of autism are embedded in various biomedical, social, psychological, economic, and cultural constructs. Understanding parental explanatory models can help demystify autism, and foster a mutually trusting, collaborative approach for the child's total care.

\section{Objectives}

The general objective of the study was to determine the association between parental perceptions of autism and their health-seeking behaviors. Specific objectives were to explore parents' understanding of autism, identify parental or child factors associated with perceptions on autism, illustrate the timeline from symptom recognition, age of initial consult, up to formal diagnosis, and determine the most common presenting symptoms that parents recognize in children diagnosed with autism.

\section{Methods}

\subsection{Study design}

This was a cross-sectional study conducted in two phases. The first phase involved development of a valid and reliable questionnaire. The second phase was a survey on parents' perceptions on autism and their health-seeking behaviors.

\subsection{Study population and setting}

A convenience sample of parents were recruited through institutions within Metro Manila - parent organizations, schools, clinics, and therapy centers -involved in the care of children with autism, during the period from June to August 2013.

\subsubsection{Inclusion criteria}

Participants of this study were parents living with their child/ children aged 2-6 years old who has/have been previously diagnosed with autism by a child development specialist. 


\subsubsection{Exclusion criteria}

Parents of children diagnosed with other pervasive developmental disorders were excluded from the study.

\subsection{Data collection}

\subsubsection{Phase 1}

Both qualitative and quantitative methods were utilized during this phase. Key informant interviews (KIIs) were conducted with two developmental pediatricians and one parent who was the president of the University of the Philippines College of Allied Medical Professions (UP CAMP) chapter of the Autism Society of the Philippines (ASP). Two focus group discussions (FGDs) were conducted. The first FGD comprised of parents of children with autism being seen at the Development and Behavioral Studies Unit of the Philippine General Hospital. The second FGD was attended by parent members of the ASP.

Data from the KIIs and FGDs were utilized to generate a valid and reliable questionnaire. Reliability was tested on 99 parent-respondents.

\subsubsection{Phase 2}

The questionnaire generated from phase 1 was administered to 41 parents of children with autism, ages 2-6. A sample of 33 parents of children with autism was required to estimate the mean age at diagnosis of autism with a 1-year margin of error at $95 \%$ confidence level, and assuming a standard deviation of 2.9 years. The mean age at diagnosis was used in the computation of the sample size because early diagnosis and intervention is one of the most crucial points in the management of children with autism.

\subsection{Statistical analysis}

Statistical analyses were performed using StataCorp. 2011. Stata Statistical Software: Release 12. College Station, TX: StataCorp LP. Thematic analysis was performed on qualitative data. Pearson's correlation and Cronbach's alpha were utilized during reliability testing of the questionnaire. Descriptive statistics were reported in means and proportions. Associations between categorical variables were tested using Chisquare statistic. For continuous variables, correlation analyses were done.

\subsection{Ethical considerations}

Written informed consent was obtained from all participants. They were assured of confidentiality, privacy and anonymity. Recruitment was done by investigators who had no professional relationship with the participants.

\section{Results}

\subsection{Reliability of questionnaire}

The 22 items in the scale on parental perceptions on autism were grouped into four general domains: (1) initial attitudes towards autism, (2) perceived parental psychosocial factors associated with autism, (3) perceived parental biologic causes
Table 1 - Demographic characteristics of parent respondents in the focus group discussions and survey.

\begin{tabular}{lcl} 
& $\begin{array}{c}\text { Focus group } \\
\text { discussions } \\
(n=15)\end{array}$ & $\begin{array}{l}\text { Survey } \\
(n=41)\end{array}$ \\
\hline Age in years (mean $\pm \mathrm{sd})$ & $39 \pm 7$ & $36 \pm 6$ \\
$\%$ female & $9 / 15(60 \%)$ & $34 / 41(83 \%)$ \\
$\%$ married & $13 / 15(87 \%)$ & $30 / 41(73 \%)$ \\
$\%$ employed & $7 / 15(47 \%)$ & $23 / 41(56 \%)$ \\
Number of children with autism: & & \\
1 & 8 & 41 \\
2 & 1 & 0 \\
\hline
\end{tabular}

of autism, and (4) perceived child-related factors associated with autism. A negative correlation in one item was accounted for by a positive statement (compared to others that were negatively stated), and hence was recoded. In the final questionnaire, all 22 items were retained.

\subsection{Demographic characteristics}

There were 3 respondents for the KIIs, 15 (6 parent-pairs and 3 parents who attended without their partners) for the FGDs, and 41 for the survey. The demographic characteristics of the parent respondents in the FGDs and survey are shown in Table 1 . The mean age was 39 years for the FGD group and 36 years for the survey group. Majority of the respondents in both groups were female and married. Roughly, half of the respondents in both groups were employed. There was one respondent in the KII and one in the FGD who had two children with autism.

The parent-respondents represented a total of 10 children with autism in the FGD group and 41 children in the survey group (Table 2).

\subsection{General knowledge and parental perceptions on autism}

\subsubsection{Qualitative analysis from KIIs and FGDs}

Since two of the respondents in the KIIs were child development specialists and one was a highly informed parent, they all had scientific views about autism. They defined autism as a triad of conditions involving impairments in communication and social skills, as well as restricted, repetitive, and stereotyped patterns of behavior. They claimed that no specific

Table 2 - Profile of children with autism of parent

respondents in the focus group discussions and survey.

\begin{tabular}{lll} 
& \multicolumn{1}{c}{$\begin{array}{c}\text { Focus group } \\
\text { discussions } \\
(n=10)\end{array}$} & \multicolumn{1}{c}{$\begin{array}{c}\text { Survey } \\
(n=41)\end{array}$} \\
\hline $\begin{array}{l}\text { Age in months } \\
(\text { mean } \pm \text { sd) }\end{array}$ & $75.43 \pm 33.74$ & $60.73 \pm 17.45$ \\
$\%$ male & $7 / 10(70 \%)$ & \\
Birth rank & $5 / 10(50 \%)$ firstborn & $36 / 41(87.8 \%)$ \\
& $21 / 41(53 \%)$ \\
Fetal maturity & $8 / 10(80 \%)$ term & firstborn \\
Mode of delivery & $7 / 10(70 \%)$ & $25 / 41(96 \%)$ term \\
& normal delivery & normal delivery \\
\hline
\end{tabular}


cause has been directly linked to autism, nor is there a cure for the condition, to date. However, they all agreed that early intervention has been shown to produce optimum results for children with autism.

During the FGDs, the parents were asked about their initial thoughts and perceptions about their child's behavior. Their answers revolved around the following: they were initially afraid to be told that their child had autism, many were in denial, other parents blamed one another, and many were hoping for their children to catch up with others. The parents had varied views about possible causes of autism. Some of the answers given were hereditary, old age of one or both parents, absence of one parent while growing up, maternal stress or drug intake during pregnancy, vaccines particularly MMR, short time interval between birth of two children, and mercury content of food. The parents were still generally confused as to how their child acquired the condition.

Since the respondents of this study were recruited through institutions/centers catering to children with autism, they had access to various types of interventions, which included one or a combination of the following: speech, occupational or physical therapy, special education, medicines, and developmental monitoring. The parents had mixed responses with regard to satisfaction with these interventions. Some noted marked improvements in their child's behavior while others only noted minimal changes.

Many of the parents had to give up their career/work to take care of their child. They were often worried about growing old, and concerned about "who will be there to help and support them?" There were also those who clamored for better support from the government for persons with autism.

\subsubsection{Quantitative analysis from survey}

Parental perceptions on autism were elicited during the survey through 5-point Likert scale items grouped into four general domains. A mean score of greater than 4 for each item meant that they generally disagreed with that statement.

In terms of parental attitudes on autism, the parents agreed with the statements that they initially thought their child would eventually catch up with other children, they were initially in denial of the diagnosis, and were afraid of receiving an official diagnosis.

For parental psychosocial factors, parents reported that lack of finances and long waiting period prior to being seen by a specialist hindered them from seeking professional consult. They disagreed with the statements that autism can be caused by a curse or is a consequence of parental $\sin / \mathrm{s}$.

The mean scores of the respondents were neutral in terms of parental biologic factors, which can cause autism, i.e. autism is hereditary, eating mercury-contaminated food by the mother during pregnancy, maternal stress, illness or trauma during pregnancy, and late age of one or both parents.

In the same manner, responses were also neutral for statements on child-related factors, which can cause autism, i.e. complications acquired during and after childbirth, vaccination in children, childhood illness or trauma before 1 year of age, and intake of certain food by the child.

\subsection{Parental health-seeking behaviors}

\subsubsection{Qualitative analysis from the FGDs}

During the FGDs, the more commonly observed initial symptoms of autism were no speech or delayed speech, lack of interaction, nonresponse when called, playing alone, and lack of eye contact. Parents, or close family and friends, were the first to notice most of the signs of autism. Their first initial professional consult was with a general pediatrician and the children were mostly aged 2 at the time of first consult. Most of the children underwent hearing tests to rule out a hearing defect. Some parents were unable to bring their child immediately to a specialist for the following reasons: lack of finances, lack of knowledge on who to consult, or a long waiting time (sometimes as long as 6 months) for an appointment with a developmental pediatrician.

\subsubsection{Quantitative analysis from survey}

From the survey on 41 parents, the first recognized impairments in their child with autism were qualitative impairment in social interaction (54\%), qualitative impairment in communication (34\%), and restrictive, repetitive stereotyped patterns of behavior (12\%).

Family members/relatives were usually the first persons to recognize the child's symptoms (78\%), followed by friends or neighbors (8\%), teachers (7\%), and doctors $(7 \%)$.

After recognition of symptoms, $34 \%$ of the parents each initially sought the opinion of a general or developmental pediatrician. There were parents who also consulted relatives or the internet (7\% each); teachers, neurologists, or friends $(5 \%$ each); and religious leaders (2\%).

The diagnosis of autism in their children was confirmed by developmental pediatricians in most cases (83\%), and the rest by general pediatricians (7\%), neurologists $(7 \%)$, and therapists (3\%). Majority of the parents (68\%) no longer sought a second opinion.

The mean age of the children at the time the initial symptoms was recognized was 24.42 months. First professional consult was sought at a mean age of 37.56 months; hence, there was a delay of 13.15 months. The mean age at confirmation of the diagnosis is 39.39 months, which is 14.98 months after initial recognition of symptoms. The interval from first professional consult to confirmation of the diagnosis is 1.83 months (Fig. 1).

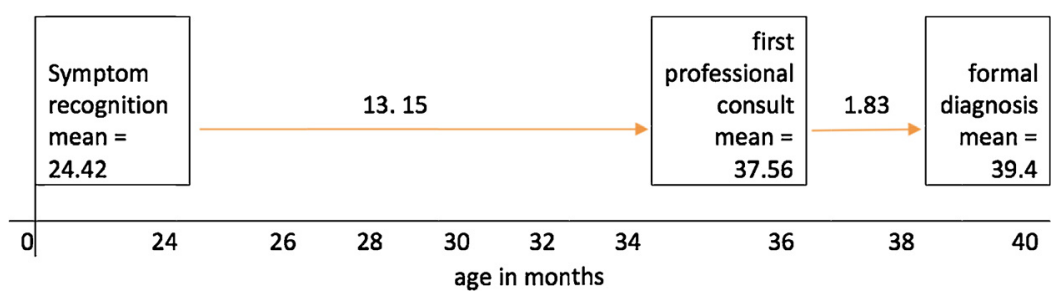

Fig. 1 - Timeline from initial recognition of symptoms to first consult and confirmation of diagnosis. 
Most of the children (54\%) were diagnosed at less than or equal to 36 months and $46 \%$ were diagnosed at more than 36 months.

There were no significant associations noted between baseline characteristics of parents or children and parental perceptions, nor between parental perceptions and their health-seeking behaviors.

\section{Discussion}

\subsection{Parental perceptions on autism}

The parental attitudes with regard to their child's attitude or initial symptoms were similar for the FGD and the survey. Most of them were in denial and had hopes that their child would eventually catch up; they were afraid of getting an official diagnosis of autism for their child. Most parents continue to hope that their child would eventually catch up with the other children. When asked about their fears, majority expressed concerns about the care of their children in their old age.

Parents in the study generally recognize that autism is a condition that is not yet fully understood, even by the scientific community. During the FGDs, biological causes surfaced more than psychosocial causes. The views were varied since these were combinations of what they heard from other people, what they read about autism from internet sources, and what they learned from the medical practitioners they have consulted. The questionnaire was designed to ask the parents about their initial perceptions regarding autism prior to seeking consult, including what they thought about the presenting symptoms. However, parents' recall may be tainted with their current knowledge of autism. The views expressed by the participants may not be the same for the general public whose children have yet to be diagnosed.

In the qualitative survey, most responses were neutral they neither agreed nor disagreed. This reflects the uncertainty that prevails in their minds with regard to the etiology of autism. Despite the uncertainty in the etiology of autism, parents were in strong agreement that autism is not due to a curse nor a consequence of parental $\sin / \mathrm{s}$. Parents in the study may no longer bear the same stigma of the parents in the past, where developmental and mental handicaps were blamed on faulty parenting styles or parental debts for their infractions. Majority of the parents in the FGD and the survey have a general idea that genes and the environment play a role in the phenotypic variability of autism.

The present study showed that parents have different sources of information regarding health. Filipinos generally value the opinion of relatives and friends although majority of the respondents reported to a professional right away. A new and readily available source of information for parents nowadays is the internet. Parents recognize the wealth of information available at their fingertips, so much so that a few parents in the study were able to reliably suspect autism in their child even before a professional consult.

\subsection{Parental health-seeking behaviors}

Majority of parents in both the FGD and the survey noted impairment in social interaction and in communication as the initial symptoms of their children, majority of which were noted by family members and relatives. Impairment in social communication is more compatible with past researches ${ }^{7}$ than the result from the survey, which suggested impairment in social skills (54\%) as the more common presenting symptom. Since the lack of appropriate language skills is usually a more obvious symptom, this new finding may suggest that parents may now be more keen in noting more subtle problems like poor social skills.

Majority of the parents in the FGD group sought the opinion of general pediatricians for their children's symptoms.

Initial symptoms were recognized at around 2 years old. First professional consult was sought at a mean age of 2.9 years in the FGD and 37.56 months or 3.13 years in the survey. There was a delay of roughly 3 months in the FGD group and 13.15 months in the survey group. The mean age at confirmation of the diagnosis in the survey is 39.39 months, which is 14.98 months after initial recognition of symptoms. This was mostly done by developmental pediatricians. Much of the delay was in the initial consult. Surprisingly, the authors did not see a long waiting period between the initial consult and confirmation of diagnosis in the survey. In the survey, the time difference between the initial consult and confirmation of the diagnosis was only 1.83 months. General pediatricians, as well as teachers, may also have a lower threshold for referral to a developmental pediatrician. This trend supports the call from many stakeholders to facilitate early diagnosis. Some parents in the FGD groups, however, were unable to bring their child immediately to a specialist due to lack of finances, lack of knowledge on who to consult, or a long waiting time for an appointment with a developmental pediatrician.

There was a trend for earlier initial consult in both the FGD and survey groups. This implies several things: (1) parents are more aware of and are more open to reporting developmental concerns, (2) heightened societal awareness has made it easier for parents, clinicians, and other adults to identify problematic behaviors in children, (3) families may have better access to diagnostic services in the recent years compared to the past decade. There is an apparent drop in the age of diagnosis of these children, compared to previous studies. ${ }^{7}$ This pattern was also reported by Fountain et al. in their study that showed a decline in the median age of diagnosis for autism, from 4.4 years in 1992 to 3 years in $2001 .^{13}$

Many of the parents in this study were open in sharing, were resourceful, empowered, and actively engaged in their child's care. The researchers also observed the relevance of having a support group for families, such as the ASP, which has become a venue for social and educational support for families living with autism.

The results of this study showed some surprisingly encouraging trends. Parents, particularly those whose children are already enrolled in schools and therapy centers, are more aware about the intricacies of autism. They tend to disagree with previous myths about autism. There was a notable tendency for earlier diagnosis among the urban 
parents in the study. Despite this, however, the mean age at formal diagnosis is still a little older than the recommended age of 36 months or less. Indeed, there is still much room for improvement in terms of timing of diagnosis. It is important to note at this point, however, that early diagnosis does not necessarily equate to early intervention. Future studies focusing on the timing of intervention among children with autism in the Philippines would yield relevant information.

This study was able to capture parents whose children were already receiving active interventions for their condition, and thus possibly accounting for the promising trends mentioned earlier. The researchers recommend actively searching out those children (and their parents) who are already exhibiting symptoms of autism but have not yet entered the healthcare system. This population might reveal an entirely different story and possibly highlight other areas of concern. The work for children with autism is far from finished. There is still much need for collaborative efforts among different sectors for the welfare of this special population.

\section{Conflicts of interest}

The authors have none to declare.

\section{REFEREN C ES}

1. Reyes A, Herrin J. A 5-year review of referrals to the developmental pediatrics section of a major tertiary hospital. Acta Medica Philippina. 2009;43(3):12-17.
2. Carbone P, Davis T, Farley M. Primary care for children with autism. Am Fam Physician. 2010;81(4):454-460.

3. Volkmar F, Chawarska K. Autism in infancy and early childhood. Annu Rev Psychol. 2005;56:315-336.

4. Wiggins L, Balo J, Rice C. Examination of the time between first evaluation and first autism spectrum diagnosis in a population-based sample. J Deu Behav Pediatr. 2006;27:79-87.

5. Daley C. From symptom recognition to diagnosis: children with autism in urban India. Soc Sci Med. 2004;58(7):1323-1325.

6. Pierce K, Carter C, Weinfeld M. Detecting, studying, and treating autism early: the one-year well baby check-up approach. J Pediatr. 2011;159:458-465.

7. Avendaño EL, Berroya MAG, Ortiz MH, et al. A profile of pervasive developmental disorders. Philipp J Neurol. 1998.

8. Moral-Valencia M, Tanglao-Salazar N, De Sagun R, et al. Factors implicated in delayed consultation of children with neurodevelopmental disabilities. St Tomas J Med. 2004;52 (1):16-23.

9. Shattuck P, Durkin M, Maenner M, et al. Timing of identification among children with autism spectrum disorder. J Am Acad Child Adolesc Psychiatry. 2009;48:474-483.

10. Quilendrino MI. Screening and evaluation for early language delay in children 12 to 36 -months old in an urban community health center. Philippine General Hospital, October 2011, unpublished.

11. Gray D. Lay conceptions of autism: parents' explanatory models. Med Anthropol. 1995;16:99-118.

12. Greydanus DE, Toledo-Pereyra LH. Historical perspectives on autism: its past record of discovery and its present state of solipsism, scepticism and sorrowful suspicion. Pediatr Clin North Am. 2012;59:1-11. http://dx.doi.org/10.1016/j. pcl.2011.10.004.

13. Fountain C, King MD, Bearman BS. Age of diagnosis for autism: individual and community factors across 10 birth cohorts. J Epidemiol Community Health. 2011;65(6):503-510. http://dx.doi.org/10.1136/jech.2009.104588. 